\title{
REVIEW
}

For reprint orders, please contact: reprints@futuremedicine.com

\section{Management of poor-risk metastatic renal cell carcinoma: current approaches, the role of temsirolimus and future directions}

\author{
Camillo Porta ${ }^{*}$, , Giampaolo Tortora², James MG Larkin³ \& Thomas E Hutson ${ }^{4,5}$
}

Targeted therapies have substantially improved outcomes in metastatic renal cell carcinoma (mRCC). As expected, poor-risk patients have the worst outcomes. Temsirolimus is currently the only agent licensed for treatment of poor-risk mRCC patients. It is associated with meaningful improvements in survival and quality of life, highlighting the importance of correctly stratifying risk in $\mathrm{mRCC}$ patients so they receive optimal treatment. Currently, data for other targeted therapies in poor-risk patients are relatively sparse. Optimizing outcomes in these patients is the subject of ongoing research, including studies of biomarkers and studies to elucidate the role of nephrectomy and neoadjuvant targeted therapy in poor-risk mRCC patients. The impacts of novel combinations including temsirolimus have also been explored to further improve outcomes.

First draft submitted: 21 September 2015; Accepted for publication: 9 November 2015; Published online: 26 November 2015

Renal cell carcinoma (RCC) represents the majority of renal cancer cases, $25 \%$ of whom present with locally invasive or metastatic disease [1]. In addition, approximately a third of patients with RCC who undergo radical nephrectomy will go on to develop distant metastases [2]. Risk profiling can be utilized to predict prognosis and survival in patients with metastatic RCC (mRCC). The most widely used risk assessment model, the Memorial Sloan-Kettering Cancer Center (MSKCC) criteria, assesses prognosis based on five risk factors (Table 1) [3-6], with patients considered 'favorablerisk' if no risk factors are present, 'intermediate risk' if they exhibit one or two risk factors and 'poor risk' if they have three or more risk factors [4]. The MSKCC criteria were developed prior to the availability of targeted therapies for the treatment of RCC. More recently, a slightly modified version of the MSKCC criteria was proposed by Heng et al. [3] in a study of mRCC patients treated with VEGF-targeted agents; the Heng criteria, which include the MSKCC criteria minus lactate dehydrogenase (LDH) serum levels and two additional risk factors (neutrophil and platelet concentrations > upper limit of laboratory's reference range [Table 1]) [3-6] have subsequently been validated [5]. Current treatment recommendations [7-10] are based on these risk assessment models. Approximately $20 \%$ of mRCC patients are categorized as poor risk according to the MSKCC criteria [4] although, when the Heng criteria were recently applied to 1028 previously unanalyzed mRCC patients, $30 \%$ were identified as poor risk [5].

\section{KEYWORDS}

- biomarkers • metastatic renal cell carcinoma - nephrectomy • poor prognostic risk $\bullet$ RCC - temsirolimus 
Table 1. Prognostic criteria to predict survival in patients with advanced renal cell

carcinoma: Memorial Sloan-Kettering Cancer Center and Heng criteria.

\begin{tabular}{|c|c|}
\hline Risk factors & Threshold value \\
\hline \multicolumn{2}{|l|}{ Risk status (number of risk factors): } \\
\hline - Low & 0 \\
\hline - Intermediate & $1-2$ \\
\hline- High & $\geq 3$ \\
\hline \multicolumn{2}{|l|}{ MSKCC } \\
\hline KPS & $<80 \%$ \\
\hline Time from diagnosis to start of systemic treatment & $<12$ months \\
\hline Hemoglobin & $<$ Lower limit of laboratory's reference range \\
\hline Lactate dehydrogenase & $\begin{array}{l}>1.5 \times \text { the upper limit of laboratory's reference } \\
\text { range }\end{array}$ \\
\hline Corrected serum calcium & $>10.0 \mathrm{mg} / \mathrm{dl}(2.4 \mathrm{mmol} / \mathrm{l})$ \\
\hline \multicolumn{2}{|l|}{ Heng criteria } \\
\hline KPS & $<80 \%$ \\
\hline Time from diagnosis to start of systemic treatment & $<12$ months \\
\hline Hemoglobin & $<$ Lower limit of laboratory's reference range \\
\hline Corrected serum calcium & $>10.0 \mathrm{mg} / \mathrm{dl}(2.4 \mathrm{mmol} / \mathrm{l})$ \\
\hline Neutrophils & >Upper limit of laboratory's reference range \\
\hline Platelets & >Upper limit of laboratory's reference range \\
\hline \multicolumn{2}{|c|}{$\begin{array}{l}\text { 'Modified in temsirolimus Phase III ARCC trial [11] to include a KPS of } 60 \text { or 70, and with the addition of metastases in multiple } \\
\text { organs; different MSKCC criteria (low hemoglobin, high corrected calcium, and low performance status [6]) used to assess } \\
\text { prognostic risk for second-line everolimus (RECORD-1) [12] and axitinib (AXIS) [13] trials. } \\
\text { KPS: Karnofsky performance status; MSKCC: Memorial Sloan-Kettering Cancer Center; RCC: Renal cell carcinoma. } \\
\text { Data taken from [3-6]. }\end{array}$} \\
\hline
\end{tabular}

Anti-angiogenic therapies targeting VEGF and the VEGF receptor (VEGFR), including sunitinib, sorafenib, bevacizumab plus IFN- $\alpha$, pazopanib and axitinib, have substantially improved outcomes in patients with mRCC in recent years [13-17]. However, the benefits of these targeted therapies have been demonstrated largely in patients with favorable- or intermediate-risk mRCC [7-9].

The mTOR inhibitor, temsirolimus (Torisel ${ }^{\circledR}$, Pfizer Inc., NY, USA), is currently the only agent licensed for the treatment of patients with poorrisk mRCC. Approval of temsirolimus was based on the results of the Phase III, randomized, multicenter ARCC trial [11]. Temsirolimus is indicated for the first-line treatment of patients with advanced RCC who demonstrate at least three of six preselected prognostic risk factors [18]. There are more recent data indicating that it may also be useful as second- and later-line therapy in mRCC [19].

Despite the availability of temsirolimus, treatment options remain limited for patients with poor-risk mRCC and further trials are needed to assess the potential benefits of other targeted agents in this setting. In addition, a number of unanswered questions remain concerning the management of these patients, including the use of biomarkers to support treatment decisionmaking, and the role and timing of nephrectomy given that patients with poor-risk mRCC appear to derive only a marginal survival benefit from cytoreductive nephrectomy [20].

This review examines the current treatment algorithm for the management of patients with poor-risk mRCC, and considers available and future therapies for this patient subgroup, as well as emerging concepts and outstanding questions in the management of this disease. Also addressed are issues that may be faced in clinical practice when determining a patient's prognostic risk group, the importance of this stratification in terms of driving the treatment they receive, and the appropriate and timely management of adverse events (AEs) for optimizing treatment for individual patients.

\section{Current recommendations for}

temsirolimus in the treatment of $\mathrm{mRCC}$

Guidelines from the European Urology Association (EUA), the European Society of Medical Oncology (ESMO), the European Organization for Research and Treatment of Cancer Genito-Urinary Group (EORTC-GU) 
and the US National Comprehensive Cancer Network (NCCN) recommend temsirolimus for the first-line treatment of patients with poor-risk mRCC based on data from the Phase III ARCC study (category 1, NCCN Guidelines), and for selected patients of other risk groups (category 2B, NCCN Guidelines) (Table 2) [7-9,21-22] .

\section{Temsirolimus monotherapy in the treatment of poor-risk $\mathrm{mRCC}$}

\section{- Rationale for the efficacy of temsirolimus} in poor-risk patients

mTOR is a highly conserved serine/threonine kinase which forms a key component of intracellular signaling pathways involved in many processes, including cell growth, proliferation and responses to hypoxia [23-25]. The mTOR pathway is dysregulated in RCC, particularly in patients with clear-cell disease, high-grade tumors and tumors with features indicative of a poor prognosis [26]. Temsirolimus binds to the intracellular FKBP-12, forming a complex that inhibits mTOR signaling [27,28]. It thus blocks phosphorylation of the protein translation factors eukaryotic translation initiation factor $4 \mathrm{E}-$ binding protein (4E-BP1) and ribosomal protein $S 6$ kinase (S6K), which control protein synthesis (Figure 1) [27-31]. Furthermore, mTOR inhibition depresses hypoxia-inducible factors, which subsequently inhibits the VEGF pathway, thus impairing angiogenesis [23,24].

\section{- Efficacy \& safety of single-agent temsirolimus in poor-risk mRCC}

Following evidence of improved survival with temsirolimus in patients with cytokine-refractory mRCC in a Phase II trial [32] the multicenter Phase III ARCC study in previously untreated patients was undertaken to compare temsirolimus with IFN- $\alpha$ [11] the standard of care at that time according to the results of a Cochrane metaanalysis [33]. Patients $(n=626)$ with poor-prognosis $\mathrm{mRCC}$ were randomized to one of the three treatment groups: temsirolimus $25 \mathrm{mg} /$ week, IFN- $\alpha 3$ million units (MU; raised to $9 \mathrm{MU}$ and then $18 \mathrm{MU}$ if tolerated) three-times weekly, or the combination of temsirolimus $15 \mathrm{mg} /$ week with IFN- $\alpha 6 \mathrm{MU}$ (starting dose $3 \mathrm{MU}$, raised to $6 \mathrm{MU}$ if tolerated) three-times weekly. The study employed modified MSKCC criteria; poor prognosis was defined as the presence of at least three of the following risk factors: serum $\mathrm{LDH}$ $>1.5 \times$ the upper limit of normal (ULN), hemoglobin <lower limit of normal (LLN), corrected serum calcium $>10 \mathrm{mg} / \mathrm{dl}$, time from initial diagnosis to randomization $<12$ months, Karnofsky performance status $60-70 \%$ and metastases in multiple organs [11].

Temsirolimus monotherapy significantly prolonged overall survival (OS), the primary study end point, relative to IFN- $\alpha$ (hazard ratio $[\mathrm{HR}]: 0.73$; $95 \%$ CI: $0.58-0.92$; $\mathrm{p}=0.008)$ [11]. However, OS did not differ significantly between the combination therapy group and the IFN- $\alpha$ monotherapy group (HR: 0.96; 95\% CI: 0.76-1.20; $\mathrm{p}=0.70$ ): median OS was 10.9 months in the temsirolimus group compared with 8.4 months in the temsirolimus + IFN- $\alpha$ group and 7.3 months in the IFN- $\alpha$ monotherapy group (Figure 2A) [11].

Progression-free survival (PFS), as determined by independent radiologic assessments, was prolonged in both temsirolimus treatment groups relative to IFN- $\alpha$ therapy [11]. Median PFS was 5.5 months with temsirolimus monotherapy, 4.7 months with temsirolimus + IFN- $\alpha$ and 3.1 months with IFN- $\alpha$ monotherapy. Median PFS by investigator assessment was 3.8 months with temsirolimus monotherapy, 3.7 months with the combination and 1.9 months with IFN- $\alpha$ monotherapy (Figure 2B). The shorter estimate of PFS by the site investigators reflected the inclusion of patients with symptomatic deterioration that had begun before scheduled radiologic measurements of the tumor.

The objective response rate (ORR) was 8.6\% with temsirolimus monotherapy, $8.1 \%$ with temsirolimus + IFN- $\alpha$ and $4.8 \%$ with IFN- $\alpha$ monotherapy; these differences were not statistically significant [11]. Treatment failure occurred after 3.8 months with temsirolimus monotherapy, 2.5 months with temsirolimus + IFN- $\alpha$ and 1.9 months with IFN- $\alpha$ monotherapy.

The AEs most commonly reported with temsirolimus in the ARCC study were asthenia, rash, anemia, nausea, anorexia, pain, dyspnea, hyperlipidemia, infection, diarrhea, peripheral edema, hyperglycemia and cough [11]. Most events were grade $1-2$ in severity and were managed with supportive therapy. Anemia, asthenia and hyperglycemia were the only grade 3-4 events that occurred in $>10 \%$ of patients and required dose adjustment. Mild-to-moderate rash, peripheral edema and stomatitis were more common in the temsirolimus treatment groups than in the IFN- $\alpha$ monotherapy group, while asthenia was more common in the IFN- $\alpha$ group; anemia, neutropenia and thrombocytopenia 


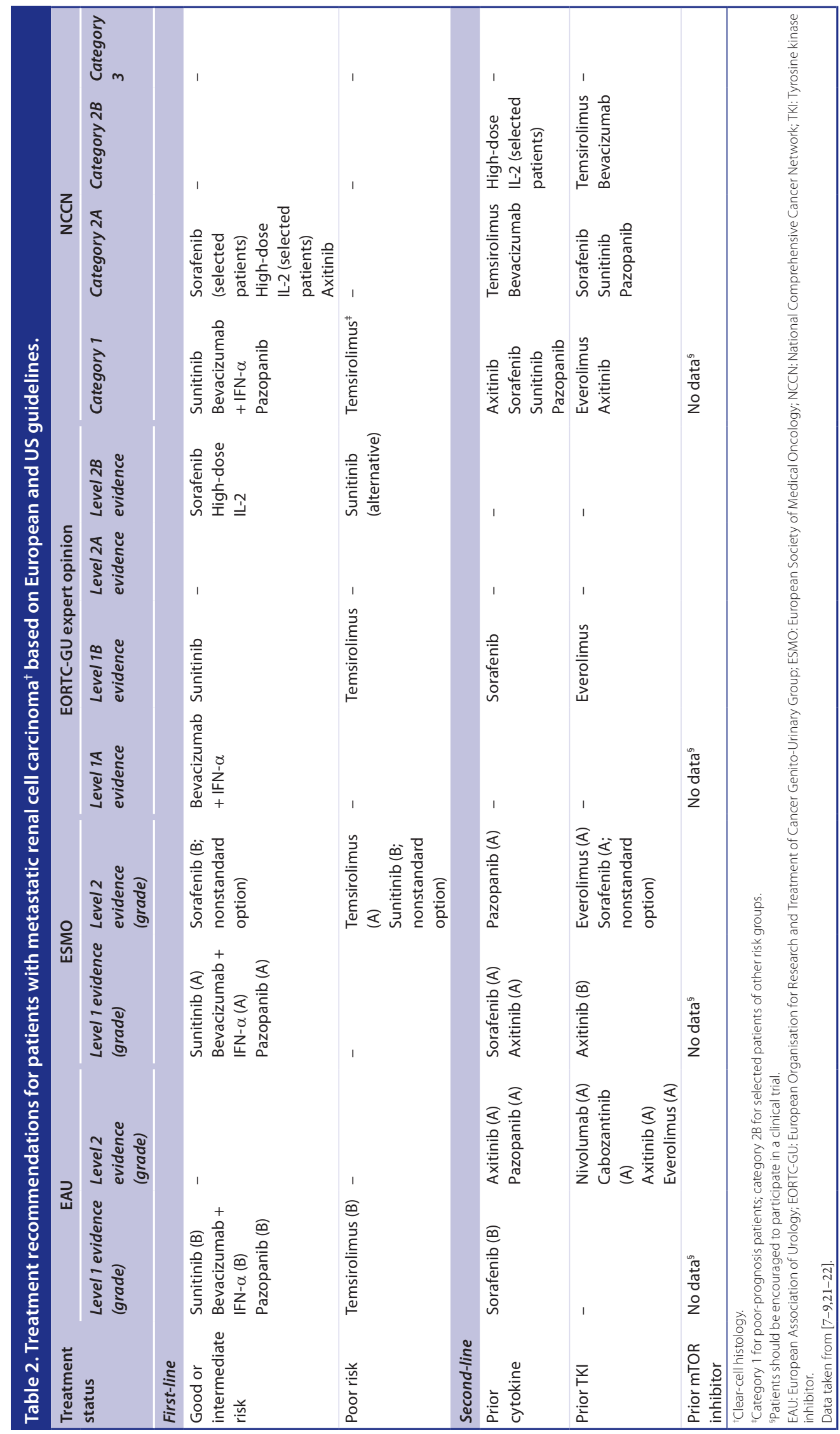




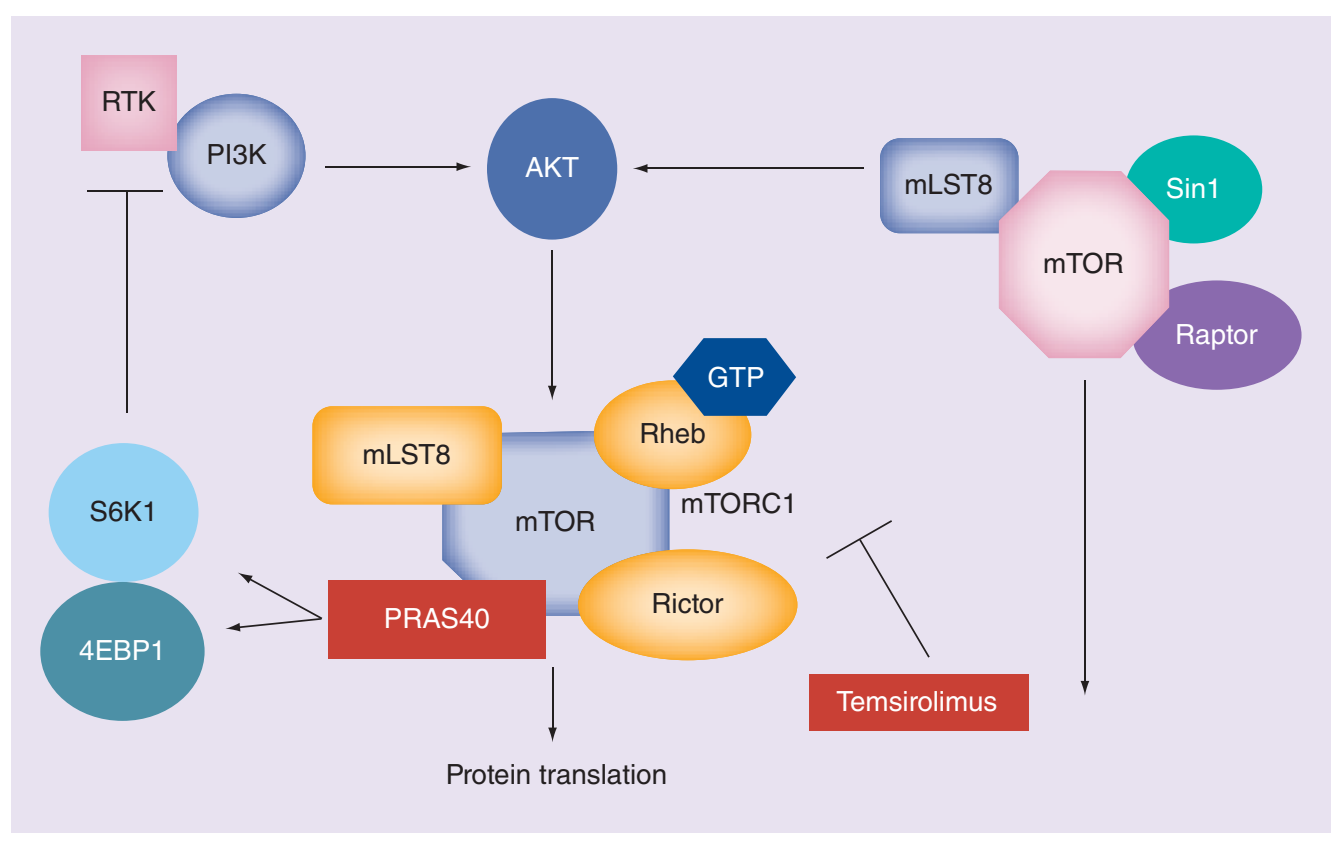

Figure 1. Signal transduction pathway involving mTOR.

occurred more frequently in the combination therapy group than in either monotherapy arm $(\mathrm{p} \leq 0.002)[11]$.

Temsirolimus treatment was also associated with an improvement in the quality of survival compared with IFN- $\alpha$ in patients in the ARCC trial. An analysis of quality-adjusted time without symptoms of progression or toxicity (Q-TWiST) demonstrated significantly longer Q-TWiST with temsirolimus monotherapy compared with IFN- $\alpha$ monotherapy (mean: 7.0 vs 5.6 months, respectively; $\mathrm{p}=0.001$ ); mean Q-TWiST in the combination therapy group (6.1 months) did not differ significantly from the IFN- $\alpha$ monotherapy group $(p=0.35)$ [34].

Analyses of data from subgroups in the ARCC trial suggested temsirolimus was at least as effective in reducing tumor mass among patients with non-clear-cell histologies as those with clear-cell RCC, and that outcomes in terms of OS and PFS were not influenced by tumor histology (clearcell vs non-clear-cell) [35]. Ongoing comparative studies will help to clarify the role of targeted therapies in the management of patients with non-clear-cell RCC [36].

Subgroup analysis of data from the ARCC trial suggested that the clinical benefit of temsirolimus in terms of OS and PFS was unaffected by prior nephrectomy [37]. A retrospective multivariate analysis found that the statistically significant OS benefit of temsirolimus monotherapy versus IFN- $\alpha$ monotherapy ( $\mathrm{p}=0.005)$ was maintained after adjusting for baseline risk factors [38]. However, other differences between the treatment cohorts may have had some impact on the study findings and should be considered. For example, patients receiving temsirolimus monotherapy experienced fewer treatment delays and dose reductions in response to grade 3-4 AEs than those receiving temsirolimus + IFN- $\alpha$, which may explain the outcomes achieved in the temsirolimus monotherapy and combination therapy groups (i.e., longer OS and PFS with temsirolimus monotherapy compared with the temsirolimus + IFN- $\alpha$ combination arm). A higher rate of grade 3-4 AEs and a higher proportion of patients discontinuing treatment due to AEs in the combination therapy group compared with the temsirolimus and IFN- $\alpha$ monotherapy arms may also have led to reduced OS in this cohort [11]. Finally, changes were made to the selection criteria after initiation of this study, and could be considered a limitation of the study design.

Results from the Phase III INTORSECT trial (NCT00474786), which compared the efficacy and safety of temsirolimus $(\mathrm{n}=259)$ with that of sorafenib ( $\mathrm{n}=253)$ as second-line treatment after first-line sunitinib, are also now available. In an analysis that included patients of varying MSKCC risk, the increased PFS seen with temsirolimus relative to sorafenib was not significant (median PFS: 4.3 vs 3.9 months, respectively [HR: 0.87; 95\% CI: 0.71-1.07; p = 0.19]). 


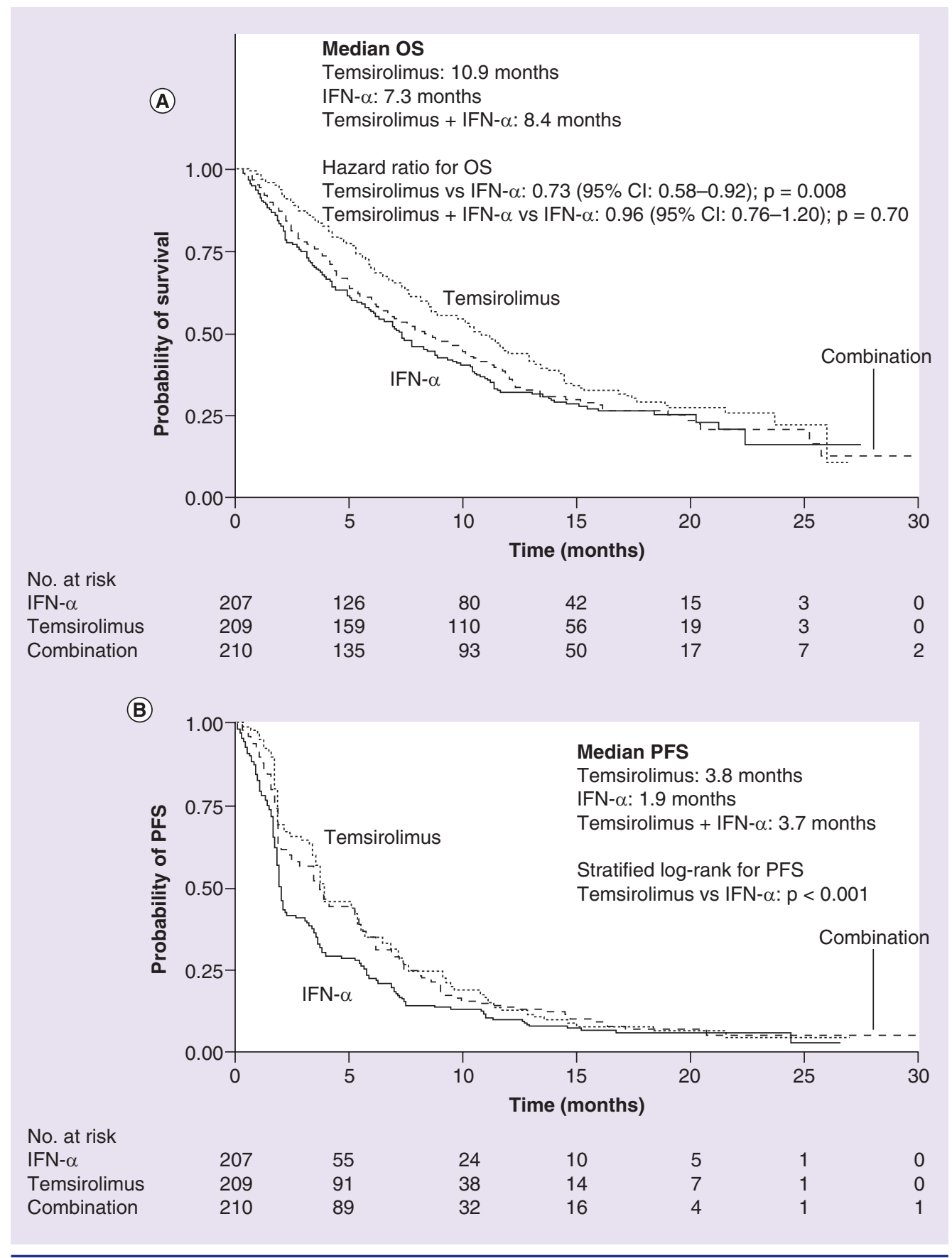

Figure 2. Kaplan-Meier curves of (A) overall survival and (B) progression-free survival in the Phase III, randomized, multicenter trial of temsirolimus with or without IFN- $\alpha$ in patients with metastatic renal cell carcinoma.

OS: Overall survival; PFS: Progression-free survival.

Reproduced with permission from [11] ๔ 2007 Massachusetts Medical Society.

Analysis of patients in the MSKCC poor-risk subgroup $(n=63)$ in which HR for PFS favored temsirolimus (HR: 0.68; 95\% CI: 0.38-1.23) was consistent with that of the overall population. Median OS was significantly longer with sorafenib (16.6 months) than with temsirolimus in the overall population (12.3 months; HR: 1.31; 95\% CI: $1.05-1.63$; $\mathrm{p}=0.01)$. The HR for OS also favored sorafenib in MSKCC poorrisk patients; however, the difference was not statistically significant (HR: $1.37 ; 95 \% \mathrm{CI}$ : 0.81-2.32) [39]. 
Recently reported data from a German multicenter registry (STAR-TOR) also support the use of temsirolimus in poor-risk mRCC patients; patients with poor-risk $\mathrm{mRCC}$ receiving firstline temsirolimus had a median PFS and OS of 3.2 and 7.3 months, respectively, and treatment was generally well tolerated [40].

\section{Identification of poor-risk patients}

Current treatment recommendations in mRCC are based on prognostic risk group. Correct risk profiling of the individual patient is therefore a key determinant in their overall management. Risk assessment models, such as the MSKCC and Heng criteria [3-6], are available to help the clinician determine prognostic group and have been widely utilized in clinical trials. However, in clinical practice, stratification of a given patient may differ according to the risk criteria applied. For example, a patient may have two of the features specified by the MSKCC criteria for an intermediate-risk score (time from diagnosis to first treatment $<1$ year and hemoglobin $<\mathrm{LLN}$ ), but may also be classified as poor risk according to the criteria used in the temsirolimus ARCC study if the presence of multiple metastatic sites is considered. This patient could thus be assigned to either a poor-risk or intermediate-risk category, and the treatment they receive would differ accordingly. For example, temsirolimus would be indicated for the firstline treatment of a poor-prognosis patient, but sunitinib may be a reasonable alternative if the patient has an intermediate prognosis.

Therefore, identification of poor-risk patients may not be straightforward (notably, poor risk is often not associated with a poor performance status), and clinicians need to be aware that different risk criteria may lead to the stratification of a given patient into a different risk group and a different treatment strategy. In addition, recent research indicates that poor-risk patients may not be a homogeneous group with similar outcomes [41].

Optimizing outcomes in poor-risk patients As treatment options for patients with poor-risk mRCC are currently limited, it is important to optimize outcomes with available therapies. A key element of treatment optimization in patients receiving temsirolimus is an effective physician-patient relationship involving appropriate education on the identification of AEs and their management prior to treatment initiation.
Through close monitoring and prompt and effective AE management, most patients are able to receive most of the planned temsirolimus dose, as demonstrated in the Phase III trial [11]. Treatment should be continued until the patient is no longer achieving clinical benefit from therapy or until the onset of unacceptable toxicities [18]. Close patient monitoring and early intervention are recommended to identify and manage AEs effectively [42].

Temporary interruption of temsirolimus or dose reduction are indicated for grade $\geq 3$ AEs [42,43], which are generally considered rare events. Following resolution of toxicities to grade $\leq 2$ severity, temsirolimus can be reintroduced at a lower dose [43].

\section{Temsirolimus combination therapies to optimize outcomes in $\mathrm{mRCC}$, including patients at poor prognostic risk}

Although the combination of temsirolimus + IFN- $\alpha$ was no more effective than temsirolimus monotherapy in patients in the ARCC study [11], a number of combination therapies incorporating temsirolimus have been under investigation in mRCC, including patients at poor prognostic risk (although these patients are a small percentage of the overall populations).

The recent Phase II TORAVA study compared the efficacy of first-line temsirolimus plus bevacizumab versus sunitinib versus bevacizumab + IFN- $\alpha$ in mRCC [44]. The study yielded disappointing results, demonstrating lower clinical activity and higher toxicity than anticipated, mirroring data from studies of everolimus in combination with bevacizumab, including the Phase II RECORD-2 study [45,46]. However, the unexpected negative results in the TORAVA study may be related to patient selection, as suggested by a surprisingly long median PFS in the bevacizumab + IFN- $\alpha$ arm (16.8 months; 95\% CI: 6.0-26.0) compared with sunitinib monotherapy (8.2 months; 95\% CI: 5.5-11.7) [44].

The Phase IIIb INTORACT study comparing temsirolimus + bevacizumab versus bevacizumab + IFN- $\alpha$ as first-line therapy in advanced mRCC patients did not meet its primary end point of increased PFS in any risk subgroup. Across all patients, median PFS was 9.1 months with bevacizumab + temsirolimus and 9.3 months with bevacizumab + IFN- $\alpha$ (HR: 1.1; 95\% CI: $0.9-1.3 ; \mathrm{p}=0.8)[47]$. These data provide a further comparison with the unexpectedly long 
PFS attained with bevacizumab + IFN- $\alpha$ in the TORAVA study [44].

The Eastern Cooperative Oncology Group (ECOG)-American College of Radiology Imaging Network (ACRIN; E2804) BeST study was a randomized Phase II trial comparing four treatment arms (bevacizumab monotherapy, temsirolimus + bevacizumab, sorafenib + bevacizumab and temsirolimus + sorafenib) in mRCC (NCT00378703) [48]. At randomization, patients were stratified according to modified MSKCC risk category and use of prior cytokine or vaccine therapy. Among eligible treated patients, median PFS $(90 \%$ CI) was 7.5 months (5.8-10.8) for bevacizumab alone versus 7.6 months (6.7-9.2) for bevacizumab + temsirolimus, 9.2 months (7.5-11.4) for bevacizumab + sorafenib, and 7.4 months (5.6-7.9) for sorafenib + temsirolimus, corresponding to HR (90\% CI) values of 1.01 (0.77-1.33), 0.89 $(0.68-1.17)$ and $1.07(0.82-1.41)$, respectively (all non statistically significant). Response rates were $13.2 \%$ for bevacizumab alone versus $31.6 \%$ for bevacizumab + temsirolimus $(p=0.008), 30.4 \%$ for bevacizumab + sorafenib $(p=0.009)$ and $20.2 \%$ for sorafenib + temsirolimus $(\mathrm{p}=0.30)$. OS, which ranged in median values from 24.3 to 28.6 months, did not differ between treatment groups. Subgroup analysis of these data will provide valuable data on the efficacy of combinations of targeted therapies in poor-risk patients, as well as those of intermediate and favorable risk.

Additional studies of temsirolimus in combination with bevacizumab in patients with advanced RCC who have progressed on anti-VEGF therapy are ongoing (NCT00782275) or actively recruiting patients (NCT01264341) [49,50].

\section{Use of therapies other than temsirolimus in poor-risk patients}

As patients with poor-risk mRCC are often excluded from clinical trials, data for this patient group are relatively limited in comparison with data for other mRCC patients. Indeed, to date, the ARCC trial [11] is the only Phase III study specifically addressing targeted therapy in poorrisk patients. In trials of therapies in which poor-risk patients are included, subanalyses of this subset are not always undertaken or are confounded by the low numbers of patients. Thus, the true value of these agents in this patient population is yet to be fully determined. However, some data regarding use of therapies other than temsirolimus in poor-risk patients are available and are discussed below.

Everolimus (Afinitor ${ }^{\circledR}$, Novartis International AG, Basel, Switzerland) is the only other mTOR inhibitor currently licensed for the treatment of mRCC [51]. Everolimus is recommended following the failure of $\geq 1$ prior tyrosine kinase inhibitors (TKIs) [7-9,21-22]. A PFS benefit was observed with everolimus (as second-, third- or further-line therapy) in the registration study (RECORD-1) across all MSKCC subgroups (including those with poor risk), although the comparator was placebo [12]. Poor-risk RCC patients treated with everolimus in a prospective Phase II study (POORTOR) had a median OS of 6.0 months (95\% CI: 4.2-8.6). However, grade 3-4 AEs related to everolimus were experienced by $43.5 \%$ of patients and serious AEs were reported in $35.5 \%$ of patients. These results suggest that everolimus is not a useful treatment option for these patients [52].

The guidelines recommend sunitinib, pazopanib and bevacizumab with IFN- $\alpha$ as first-line treatments for patients with low- or intermediate-risk mRCC (Table 2) [7-9]. A subanalysis of the sunitinib Phase III registration study by MSKCC risk stratification has also demonstrated that the agent has activity in the first-line treatment of poor-risk mRCC, although these data should be interpreted with caution as the analysis was performed in a small number of patients $(6 \%$ of the sunitinib-treated group vs $7 \%$ of the IFN- $\alpha$ treated group); median OS was 5.3 months with sunitinib versus 4.0 months with IFN- $\alpha$ (HR for OS: 0.660 ; 95\% CI: 0.360-1.207) [16,53].

An expanded-access trial of sunitinib, which included 1177/4543 (26\%) poor-risk patients according to modified MSKCC prognostic criteria, has also been reported. In the poor-risk subgroup, median PFS was 5.4 months $(95 \% \mathrm{CI}$ : 5.1-5.7) and median OS was 9.1 months (95\% CI: 8.4-9.7) [54]. In a retrospective analysis of poor-risk patients treated with sunitinib $(n=61)$, median PFS was 3.9 months and median OS was 6.4 months [55]. A more recent analysis of patients who received sunitinib therapy largely in general clinical practice determined a median OS of 5.9 months in poor-risk patients [56]. Use of sunitinib in conjunction with gemcitabine has showed early promise in a Phase I study; among 12 poor-risk RCC patients according to MSKCC criteria or with disease characterized histologically by sarcomatoid differentiation or Fuhrman grade 4/4 features, a 
partial response was seen in five patients and stable disease in seven patients. Sunitinib and gemcitabine were well tolerated [57]. Furthermore, poor-risk mRCC patients receiving sunitinib in combination with gemcitabine in a Phase II study achieved an ORR of $24 \%$ and a median OS of 15 months (95\% CI: 9-29) [58].

The Phase III AXIS trial compared axitinib with sorafenib in the second-line setting and included 238/723 (33\%) patients with poorrisk mRCC as classified by MSKCC $[13,59]$. For all study patients, median PFS by independent radiology review committee assessment (the primary end point) was 6.7 months with axitinib and 4.7 months with sorafenib (HR: 0.665; 95\% CI: 0.544-0.812; p < 0.0001) [13]. However, median OS was not significantly different between treatment groups (20.1 months with axitinib and 19.2 months with sorafenib [HR: $0.969 ; 95 \%$ CI: $0.800-1.174 ; \mathrm{p}=0.3744$ ]) [59]. In a subanalysis of data according to MSKCC risk score for the overall trial population (i.e., axitinib and sorafenib arms combined), median OS was 10.6 months (95\% CI: 9.1-12.1) in the poor-risk group, compared with 31.6 months (95\% CI: 27.2-not estimable) and 21.4 months (95\% CI: 18.9-24.0) in the favorable- and intermediate-risk groups, respectively [59].

The AVOREN trial compared the efficacy of bevacizumab in combination with IFN- $\alpha$ with IFN- $\alpha$ plus placebo and included 54/649 (8\%) patients with poor-risk mRCC as classified by MSKCC. In these patients, the median OS of 6.0 months for the combination arm was not significantly different to that achieved in the IFN- $\alpha$ plus placebo arm (5.1 months; HR: 0.85; 95\% CI: $0.49-1.47 ; \mathrm{p}=0.559)[15,60]$. The efficacy of the same combination was assessed in a further trial (CALGB 90206), which included 75/732 (10\%) poor-risk patients by MSKCC classification. The poor-risk cohort had a median PFS of 3.3 months (95\% CI: 2.2-4.7 months) for bevacizumab plus IFN- $\alpha$ versus 2.6 months (95\% CI: $1.6-3.1$ months) for IFN- $\alpha$ monotherapy [61]. For these poor-risk patients, median OS was not significantly different between treatment groups and was 6.6 months $(95 \% \mathrm{CI}$ : 5.9-8.9 months) with bevacizumab plus IFN- $\alpha$ and 5.7 months (95\% CI: 4.4-9.2 months) with IFN- $\alpha$ alone $(\mathrm{p}=0.244)$ [62].

The TIVO-1 trial compared tivozanib with sorafenib as first-line therapy. In analyses of all patients $(\mathrm{n}=517)$, tivozanib was superior to sorafenib in terms of median PFS (11.9 vs 9.1 months; HR: 0.797; 95\% CI: 0.639-0.993; $p=0.042)$. Tivozanib showed a consistent advantage to sorafenib in all patient subgroups except those with poor risk in whom sorafenib was associated with longer PFS, although this group comprised only 27 patients [63].

Finally, an open-label Phase III trial evaluated the TKI cabozantinib versus everolimus in mRCC patients who had progressed after VEGF-targeted therapy $(n=658)$ [64]. Median PFS, the primary end point, was 7.4 versus 3.8 months, respectively (HR: $0.58 ; 95 \% \mathrm{CI}$ : $0.45-0.75 ; \mathrm{p}<0.001)$; OS was longer with cabozantinib than with everolimus (HR: 0.67; 95\% CI: 0.51-0.89; $\mathrm{p}=0.005)$, but was not significant. As a result, the EAU guidelines were updated to include cabozantinib for patients with prior TKI therapy [22].

\section{- Other planned/ongoing trials including poor-risk mRCC}

In addition to the reported studies of temsirolimus or other targeted therapies described above, there are further planned or ongoing trials that will include poor-risk patients. For example, a Phase II trial, which commenced in September 2012 (NCT01392183), is comparing temsirolimus with pazopanib in patients with poor-risk clear-cell mRCC [65]. The primary outcome measure will be PFS and the trial will also evaluate patient quality of life during treatment.

Use of immunotherapy in conjunction with targeted therapy has yielded promising results in mRCC patients at poor risk. A Phase II study of sunitinib + autologous immunotherapy reported a median PFS of 5.8 months and a median OS of 9.1 months in ten patients with poorrisk mRCC [66]. The Phase III ADAPT trial (NCT01582672) is ongoing and will attempt to validate these preliminary data [67].

Immune checkpoint inhibitors - antibodies that block regulatory pathways such as PD-1, PD-L1 and CTLA4 - are also undergoing evaluation for mRCC [68]. Results to date are promising, with durable responses shown, and have generated a resurgence in interest in immunotherapy [69]. In a dose-ranging Phase II study of the PD-1 inhibitor nivolumab in previously treated mRCC patients $(n=168), 25 \%$ were classified as MSKCC poor-risk and had a median OS of 12.5 months (95\% CI: 8.1-18.6 months) [70,71]. In addition, an open-label Phase III study of nivolumab versus everolimus in previously 
treated mRCC patients $(\mathrm{n}=821)$ was recently stopped early because the data monitoring committee concluded that it had met its end point, demonstrating superior OS in patients receiving nivolumab [72] and resulting in its inclusion in the updated EAU guidelines [22]; median OS was 25.0 months (95\% CI: 21.8 -not estimable) with nivolumab and 19.6 months (95\% CI: 17.6-23.1) with everolimus (HR: 0.73; 98.5\% CI: 0.57-0.93; $\mathrm{p}=0.002)$ [72].

Pazopanib has recently been approved for first-line therapy of mRCC. However, in the pivotal Phase III trial of pazopanib in this setting $(\mathrm{n}=435)$, only $14(3 \%)$ patients were classified as MSKCC poor-risk and data for this subgroup are not available [17]. In the COMPARZ trial that evaluated pazopanib versus sunitinib, several poor prognosis patients were included in the sunitinib/pazopanib arms (9\%/12\% per MSKCC and $17 \% / 19 \%$ per Heng criteria, respectively), but there was no subgroup analysis of these data [73]. Tumor biomarker analysis in this study found that increased tumor cell PD-L1, or PD-L1 plus tumor CD8positive T-cell counts, were associated with shorter OS [74], which may indicate a role for immune checkpoint inhibitors in select patients. Consequently, the FLIPPER trial, a Phase IV study to evaluate first-line pazopanib in poorrisk mRCC patients, has been undertaken and data are eagerly awaited [75]. A Spanish study of pazopanib has shown the drug to have efficacy when used first-line in patients with poor-risk, as determined by MSKCC criteria. In this patient group $(n=21)$, time to treatment failure (TTF) due to progression or toxicity was 6 months (95\% CI: 4-8 months), 1-year TTF rate was $7.4 \%$ and the 1 -year OS rate was $55 \%$; median OS has not yet been reached [76].

Finally, because many patients relapse following treatment with VEGFR and mTORC1 inhibitors, such as sunitinib and temsirolimus, respectively, due to acquired resistance, novel agents that target PI3K and/or mTORC1/2 and demonstrate antitumor activity in preclinical RCC models are in development [77].

BKM120, a PI3K inhibitor, is being investigated in several Phase I and II trials of patients with advanced solid tumors, as monotherapy or in combination with chemotherapy or other targeted agents, including a Phase I combination trial with bevacizumab in mRCC patients [77]. In a Phase I trial of patients with advanced solid tumors $(\mathrm{n}=77)$, BKM120 was well tolerated and associated with antitumor activity in $42 \%$ of patients [78,79].

BEZ235, a PI3K/mTORC1/2 inhibitor, is being investigated in a Phase I/II trial of advanced RCC patients, in combination with everolimus in a Phase I trial of patients with advanced solid tumors, including mRCC, and several Phase I or I/II trials as a single agent or in combination with chemotherapy or other targeted agents [77]. In a Phase I trial of patients with advanced solid tumors ( $\mathrm{n}=59)$, BEZ235 was well tolerated and demonstrated preliminary efficacy [80].

GDC-0980, another PI3K/mTORC1/2 inhibitor, was investigated in comparison with everolimus in a Phase II trial of mRCC patients who had progressed during/following VEGF-targeted therapy; however, median PFS, the primary end point, was significantly shorter with GDC0980 (3.7 vs 6.1 months; HR: 2.04 [95\% CI: 1.18-3.54]; $\mathrm{p}<0.01$ ) [81]. Similarly, a Phase II study of the mTORC1/2 inhibitor AZD2014 was stopped early when it failed to improve PFS compared with everolimus in VEGF-refractory mRCC patients (1.8 vs 4.6 months; HR: 2.8 [95\% CI: 1.2-6.5]; $\mathrm{p}=0.01)$ [82].

\section{Current questions in the management of}

\section{poor-risk mRCC patients}

\section{- The role of nephrectomy in the} management of poor-risk patients

In the past, cytoreductive nephrectomy was the accepted standard of care for patients with mRCC, based on improved survival in combination with IFN- $\alpha$ therapy $[83,84]$. However, despite data to suggest that the benefit of nephrectomy can be maintained in the era of targeted therapies [85], the benefit and optimal timing of nephrectomy are now in question [9]. Patients with poor-risk mRCC appear to derive only a marginal survival benefit from cytoreductive nephrectomy, unlike those with intermediateor favorable-risk disease [20]. Factors associated with death after surgery include the number of metastatic sites, symptoms at presentation, poor performance status, high tumor grade and the presence of sarcomatoid features [86]. Indeed, while a subanalysis of data from the ARCC trial showed that temsirolimus was beneficial in both nephrectomized and non-nephrectomized patients, slight reductions in OS and PFS durations were reported in temsirolimus-treated patients who had undergone nephrectomy compared with those without nephrectomy (these differences were not statistically significant) [37]. 
Treatment with targeted agents may help to shrink the primary tumor, making patients better candidates for surgery and thereby offering a greater likelihood of improved surgical outcomes. Small case series and retrospective analyses support the benefit of anti-angiogenic therapy prior to cytoreductive surgery in patients with mRCC [10], including a study of patients with mRCC of intermediate $(82 \%)$ or poor $(18 \%)$ prognostic risk [87]. In this prospective study, $84 \%$ of mRCC patients (42/50) who received bevacizumab before planned cytoreductive nephrectomy were able to undergo surgery. ORR was $12 \%$, PFS was 11 months and OS was 25.4 months; data demonstrated no statistically significant differences between risk groups for ORR, PFS or OS [84]. However, as targeted agents appear to be more effective against metastatic lesions than primary lesions [88], this strategy may still be considered experimental. Two randomized Phase III studies are currently in progress: results from the CARMENA study (NCT00930033) [89] assessing nephrectomy with sunitinib therapy versus sunitinib alone in patients with mRCC, and a European Organisation for Research and Treatment of Cancer study (NCT01099423; the SURTIME trial) [90] assessing immediate versus deferred nephrectomy in patients with mRCC treated with sunitinib, will be available in 2017 and 2016, respectively; the likely impact of these trials on the management of poor-risk patients, specifically, remains unknown.

\section{The utility of treatment biomarkers}

The identification and validation of appropriate markers of treatment outcomes may allow therapies to be targeted to those mRCC patients who are most likely to derive benefit, thus supporting treatment optimization and cost efficacy [10]. This strategy is particularly important for poor-risk patients, for whom treatment options are limited. With this goal in mind, considerable research is ongoing to identify potential treatment biomarkers that might assist physicians with therapeutic decisionmaking and support individualized therapy for patients with mRCC.

Research into potential biomarkers of temsirolimus efficacy has focused on components of the mTOR pathway, which is particularly altered in tumors with poor-prognostic features [26], although their development has not significantly progressed. Data from a Phase II trial demonstrated a positive association between phosphorylated S6 (pS6) tissue expression and response to temsirolimus $(\mathrm{p}=0.02)$, and a trend toward a positive association between pAkt expression and treatment response $(\mathrm{p}=0.07)$. Patients whose tumors did not express high levels of pS6 or pAkt did not exhibit an objective response to temsirolimus [91]; however, the promise of $\mathrm{pS6}$ as a biomarker has yet to be borne out with additional research. In a separate study of a large multi-institutional cohort of mRCC patients, mutations in the mTOR pathway genes MTOR, TSC1 or TSC2 were more common in patients with clinical benefit from everolimus or temsirolimus than in those with rapid progression [92].

Data from exploratory subgroup analyses from the Phase III trial of temsirolimus in the poorrisk setting [11] have failed to show an association between loss of the tumor suppressor gene PTEN and treatment response [93]. Similarly, there was no correlation between baseline HIF-1 $\alpha$ status and treatment effect; temsirolimus was associated with an OS and PFS benefit regardless of PTEN or HIF1 $\alpha$ status in these patients [93].

Although tissue biomarkers hold promise in mRCC, there can be several sources of variability in sample acquisition, processing, storage and analysis which hamper the development and validation of such markers [94]. Therefore, biomarkers that can be measured easily and consistently may be more useful clinically than tissue biomarkers, with their inherent potential for variability. Levels of serum LDH may represent a potential predictive biomarker for temsirolimus therapy in poor-risk patients. Indeed, in a subanalysis of the ARCC trial, temsirolimus was significantly more effective than IFN- $\alpha$ monotherapy in poor-risk patients with baseline $\mathrm{LDH}$ levels of $>1 \times$ ULN $(p<0.002)$ than in those with $\mathrm{LDH} \leq 1 \times \mathrm{ULN}$. A decline in $\mathrm{LDH}$ during therapy was prognostic for OS ( $p<0.0001)$ [95].

An emerging concept with targeted therapies is the use of pharmacodynamic parameters as predictive markers of efficacy. For example, changes in fasting glucose and triglyceride levels have been proposed as potential pharmacodynamic biomarkers of mTOR inhibition [96]. In a recent series of exploratory analyses, changes in cholesterol, triglyceride and glucose levels compared with baseline were examined as potential predictors of clinical efficacy in patients treated with temsirolimus or IFN- $\alpha$ [97]. Temsirolimus was associated with larger mean increases in 
cholesterol, triglyceride and glucose levels compared with IFN- $\alpha$ treatment, and with improved survival (OS and PFS). In addition, increased cholesterol (but not triglyceride or glucose) was associated with longer survival in temsirolimustreated patients. Notably, no additional survival advantage over IFN- $\alpha$ was found when the treatment effect of cholesterol was accounted for in multivariate analysis, suggesting that increased cholesterol accounted for the difference in survival between temsirolimus and IFN- $\alpha$.

There is a growing body of evidence supporting the value of treatment-associated AEs as biomarkers of efficacy for targeted therapies [98]. For example, the analyses discussed above suggest that the development of hypercholesterolemia may be an important predictor for clinical benefit with temsirolimus. The use of mTOR inhibitors may also be associated with the development of pneumonitis; radiographic signs of pneumonitis are more common than clinical signs [99] and have been reported in $29-46 \%$ of patients receiving temsirolimus or everolimus, including $29 \%$ of temsirolimus-treated, poor-risk patients in the ARCC trial [100]. In one analysis, patients receiving temsirolimus or everolimus who developed radiographic pneumonitis $(14 / 46 ; 30 \%)$ were less likely to have progressive disease $(2 / 14$ [14\%] patients with pneumonitis vs $18 / 32$ [56\%] patients without) and more likely to show tumor shrinkage $(-2.9 \%$ mean reduction in tumor size) during treatment than patients who did not exhibit pneumonitis $(+4.3 \%$ mean change in tumor size; $\mathrm{p}=0.002)$. In addition, stable disease was achieved in $12 / 14$ (86\%) patients with radiographic pneumonitis compared with $14 / 32$ $(44 \%)$ without $(\mathrm{p}=0.01)$ [101]. While preliminary, these data suggest that pneumonitis may be a predictive factor for outcome to therapy with mTOR inhibitors in patients with mRCC. These findings highlight the importance of prompt and accurate diagnosis of AEs and effective AE management to maintain patients on therapy to support optimal clinical benefit. However, it should also be noted that, while certain side effects of targeted therapy do appear to be associated with improved clinical outcomes, the data are derived solely from retrospective studies and there are currently no biomarkers recommended for use in clinical practice. As such, the potential biomarkers identified to date should be regarded as hypotheses-generating only until a time when these findings can be adequately validated in well-designed, prospective clinical studies.

\section{Conclusion}

Targeted therapies have resulted in substantially improved outcomes for patients with mRCC. While outcomes are worse in patients with poorrisk disease, treatment with temsirolimus has led to meaningful improvements in the duration and quality of survival in these patients, and it is currently the only agent specifically recommended and licensed for this patient group. Currently, data on the use of targeted therapies other than temsirolimus in poor-risk patients are relatively sparse. Ongoing research is expected to enhance the future management of patients with poor-risk mRCC by helping to identify predictive biomarkers, clarify the role of targeted agents as first- or second-line therapies, identify novel treatment combinations (e.g., with immunotherapy or $\mathrm{PI} 3 \mathrm{~K} / \mathrm{mTORC} 1 / 2$ inhibitors) that may enhance outcomes, and clarify the role and timing of nephrectomy in relation to treatment.

\section{Future perspective}

Correct risk profiling of individual patients will be a key determinant in the treatment strategy and overall management of patients with mRCC. Clinicians should develop an increased awareness of the different risk criteria and the impact of these on treatment decisions. As clinical trial data emerge for novel targeted agents and novel therapeutic combinations, the treatment options for patients with poor-risk mRCC are likely to increase. In the nearer term, it will be important to optimize outcomes with currently available therapies, in particular with respect to therapy and AE management. In addition, research into the role and timing of nephrectomy may also help to optimize clinical outcomes, as will studies of biomarkers of response.

\section{Disclosure}

The manuscript was prepared in accordance with international guidelines for ethical medical writing.

Financial \& competing interests disclosure

$C$ Porta has received honoraria from Pfizer, GSK, F Hoffmann-La Roche, Novartis, Bayer-Schering, Aveo and Astellas; he has also received research funding from Bayer-Schering and Novartis. G Tortora has received honoraria from Pfizer, GSK, Novartis and Bayer-Schering, and is supported by a grant from Associazione Italiana per la Ricerca sul Cancro (AIRC IG11930). JMG Larkin has had a nonremunerated consultancy role with Novartis, GSK, Pfizer, MSD, Roche and BMS, and has received institutional research funding from Novartis, Pfizer, BMS and 
MSD. TE Hutson has a consultant/advisory role with, and has received honoraria and research funding from, Pfizer, Bayer, GSK, Novartis, Aveo and Genentech. The authors have no other relevant affiliations or financial involvement with any organization or entity with a financial interest in or financial conflict with the subject matter or materials discussed in the manuscript apart from those disclosed.

Medical writing support (incorporation of author comments, copyediting and editorial assistance) was provided by
Rachel Mason at ACUMED ${ }^{\circledR}$, an Ashfield company, part of UDG Healthcare plc (Tytherington, UK), with funding from Pfizer Inc.

\section{Open access}

This work is licensed under the Creative Commons Attribution-NonCommercial 4.0 Unported License. To view a copy of this license, visit http://creativecommons.org/ licenses/by-nc-nd/4.0/

\section{EXECUTIVE SUMMARY}

\section{Background}

- Targeted therapies have substantially improved outcomes in metastatic renal cell carcinoma (mRCC) in recent years; however, the mTOR inhibitor temsirolimus is currently the only approved agent for the treatment of patients with poor-risk mRCC.

\section{Current recommendations for temsirolimus in the treatment of mRCC}

- Guidelines from the European Urology Association (EUA), the European Society of Medical Oncology (ESMO), the European Organization for Research and Treatment of Cancer Genito-Urinary Group (EORTC-GU) and the US National Comprehensive Cancer Network (NCCN) recommend temsirolimus for the first-line treatment of patients with poor-risk mRCC based on data from the Phase III ARCC study (category 1, NCCN Guidelines), and for selected patients of other risk groups (category 2B, NCCN Guidelines).

- Sunitinib is recommended as an alternative first-line treatment in poor-risk patients (level 2B evidence, ESMO and EORTC-GU Guidelines).

\section{Temsirolimus monotherapy in the treatment of poor-risk mRCC}

- In a pivotal trial, patients with poor-risk mRCC had improved outcomes when treated with temsirolimus compared with IFN- $\alpha$ or the combination of temsirolimus and IFN- $\alpha$.

\section{Identification of poor-risk patients}

- Identification of poor-risk patients may not be straightforward, and clinicians need to be aware that different risk criteria may lead to the stratification of a given patient into a different risk group and a different treatment strategy.

\section{Optimizing outcomes in poor-risk patients}

- As treatment options for patients with poor-risk mRCC are currently limited, it is important to optimize outcomes with currently available therapies, in particular with respect to therapy and adverse event management.

- A number of combination therapies, incorporating temsirolimus, have been under investigation in mRCC, including patients at poor prognostic risk.

\section{Use of therapies other than temsirolimus in poor-risk patients}

- Data for other targeted therapies in poor-risk mRCC are currently limited. However, the available data suggest that the VEGF receptor-targeting agent sunitinib has activity while the risks of another mTOR inhibitor, everolimus, appear to outweigh the benefits in this setting.

\section{Current questions in the management of poor-risk mRCC patients}

- Further research is needed to assess other targeted agents and the role of biomarkers in poor-risk mRCC patients, as well as the effect of nephrectomy, which, unlike in intermediate- or favorable-risk patients, appears to have marginal benefit in poor-risk patients.

\section{Conclusion}

- Treatment with temsirolimus has led to meaningful improvements in the duration and quality of survival in patients with poor-risk disease, and it is currently the principal agent specifically recommended and licensed for this patient group. 


\section{References}

Papers of special note have been highlighted as:

- of interest; $\bullet$ of considerable interest

1 Cohen HT, McGovern FJ. Renal-cell carcinoma. N. Engl. J. Med. 353, 2477-2490 (2005).

2 Janzen NK, Kim HL, Figlin RA et al. Surveillance after radical or partial nephrectomy for localized renal cell carcinoma and management of recurrent disease. Urol. Clin. North Am. 30, 843-852 (2003).

3 Heng DYC, Xie W, Regan MM et al. Prognostic factors for overall survival in patients with metastatic renal cell carcinoma treated with vascular endothelial growth factor-targeted agents: results from a large, multicenter study. J. Clin. Oncol. 27, 5794-5799 (2009).

4 Motzer RJ, Bacik J, Murphy BA et al. Interferon-alfa as a comparative treatment for clinical trials of new therapies against advanced renal cell carcinoma. J. Clin. Oncol. 20, 289-296 (2002).

5 Heng DY, Xie W, Regan MM et al. External validation and comparison with other models of the International Metastatic Renal-Cell Carcinoma Database Consortium prognostic model: a population-based study. Lancet Oncol. 14, 141-148 (2013).

6 Motzer RJ, Bacik J, Schwartz LH et al. Prognostic factors for survival in previously treated patients with metastatic renal cell carcinoma. J. Clin. Oncol. 22, 454-463 (2004).

7 de Reijke TM, Bellmunt J, van Poppel H et al. EORTC-GU group expert opinion on metastatic renal cell cancer. Eur. J. Cancer 45, 765-773 (2009).

8 Escudier B, Porta C, Schmidinger M et al. Renal cell carcinoma: ESMO Clinical Practice Guidelines for diagnosis, treatment and follow-up. Ann. Oncol. 25(Suppl. 3), iii49-iii56 (2014).

9 Ljungberg B, Bensalah K, Bex A et al. Guidelines on renal cell carcinoma. European Association of Urology (2015). http://uroweb.org

10 Ryan C, Jonasch E, Lara PN Jr. Evolving Standards of Care for Advanced Renal Cell Cancer. 2010 Educational Book. ASCO University (2010).

11 Hudes G, Carducci M, Tomczak P et al. Temsirolimus, interferon alfa, or both for advanced renal-cell carcinoma. $N$. Engl. J. Med. 356, 2271-2281 (2007).

- Temsirolimus monotherapy is recommended for previously untreated poor-risk metastatic renal cell carcinoma (mRCC) patients based on data from this Phase III randomized trial (ARCC) of temsirolimus versus IFN- $\alpha$.

12 Motzer RJ, Escudier B, Oudard S et al. Efficacy of everolimus in advanced renal cell carcinoma: a double-blind, randomised, placebo-controlled Phase III trial. Lancet 372, 449-456 (2008).

-• The Phase III RECORD-1 trial was the registrational study of everolimus in $\mathrm{mRCC}$ patients.

13 Rini BI, Escudier B, Tomczak P et al. Comparative effectiveness of axitinib versus sorafenib in advanced renal cell carcinoma (AXIS): a randomised Phase 3 trial. Lancet 378, 1931-1939 (2011).

14 Escudier B, Eisen T, Stadler WM et al. Sorafenib in advanced clear-cell renal-cell carcinoma. N. Engl. J. Med. 356, 125-134 (2007).

15 Escudier B, Pluzanska A, Koralewski P et al. Bevacizumab plus interferon alfa-2a for treatment of metastatic renal cell carcinoma: a randomised, double-blind Phase III trial. Lancet 370, 2103-2111 (2007).

- The Phase III AVOREN trial compared the efficacy of bevacizumab plus IFN- $\alpha$ versus placebo plus IFN- $\alpha$ in mRCC patients.

16 Motzer RJ, Hutson TE, Tomczak P et al. Sunitinib versus interferon alfa in metastatic renal-cell carcinoma. N. Engl. J. Med. 356, 115-124 (2007).

17 Sternberg CN, Hawkins RE, Wagstaff J et al. A randomised, double-blind Phase III study of pazopanib in patients with advanced and/ or metastatic renal cell carcinoma: final overall survival results and safety update. Eur. J. Cancer 49, 1287-1296 (2013).

18 Pfizer. Torisel ${ }^{\circledR}$ (temsirolimus) summary of product characteristics (2014). www.medicines.org.uk

19 Stenner-Liewen F, Grünwald V, Greil R, Porta C. Expert Rev. Anticancer Ther. 13, 1021-1033 (2013).

20 Choueiri TK, Xie W, Kollmannsberger C et al. The impact of cytoreductive nephrectomy on survival of patients with metastatic renal cell carcinoma receiving vascular endothelial growth factor targeted therapy. J. Urol. 185, 60-66 (2011).

21 National Comprehensive Cancer Network (NCCN). National comprehensive cancer network clinical practice guidelines in oncology: kidney cancer. version 3.2015. NCCN Guidelines, Fort Washington, PA, USA.
22 Powles T, Staehler M, Ljungberg B et al. Updated EAU guidelines for clear cell renal cancer patients who fail VEGF targeted therapy. Eur. Urol. doi:10.1016/j. eururo.2015.10.017 (2015) (Epub ahead of print).

23 Fingar DC, Richardson CJ, Tee AR et al. mTOR controls cell cycle progression through its cell growth effectors S6K1 and 4E-BP1/ eukaryotic translation initiation factor $4 \mathrm{E}$. Mol. Cell Biol. 24, 200-216 (2004).

24 Hudson CC, Liu M, Chiang GG et al. Regulation of hypoxia-inducible factor 1alpha expression and function by the mammalian target of rapamycin. Mol. Cell Biol. 22 , 7004-7014 (2002).

25 Schmelzle T, Hall MN. TOR, a central controller of cell growth. Cell 103, 253-262 (2000).

26 Pantuck AJ, Seligson DB, Klatte T et al. Prognostic relevance of the mTOR pathway in renal cell carcinoma: implications for molecular patient selection for targeted therapy. Cancer 109, 2257-2267 (2007).

27 Harding MW. Immunophilins, mTOR, and pharmacodynamic strategies for a targeted cancer therapy. Clin. Cancer Res. 9, 2882-2886 (2003).

28 Hidalgo M, Rowinsky EK. The rapamycinsensitive signal transduction pathway as a target for cancer therapy. Oncogene 19, 6680-6686 (2000).

29 Loewith R, Jacinto E, Wullschleger $S$ et al. Two TOR complexes, only one of which is rapamycin sensitive, have distinct roles in cell growth control. Mol. Cell 10, 457-468 (2002).

30 Sarbassov DD, Ali SM, Sengupta S et al. Prolonged rapamycin treatment inhibits mTORC2 assembly and Akt/PKB. Mol. Cell 22, 159-168 (2006).

31 Zeng Z, Sarbassov dos D, Samudio IJ et al. Rapamycin derivatives reduce mTORC2 signaling and inhibit AKT activation in AML. Blood 109, 3509-3512 (2007)

32 Atkins MB, Hidalgo M, Stadler WM et al. Randomized Phase II study of multiple dose levels of CCI-779, a novel mammalian target of rapamycin kinase inhibitor, in patients with advanced refractory renal cell carcinoma. J. Clin. Oncol. 22, 909-918 (2004).

33 Coppin C, Porzsolt F, Awa A et al. Immunotherapy for advanced renal cell cancer. Cochrane Database Syst. Rev. (1), CD001425 (2005)

34 Zbrozek AS, Hudes G, Levy D et al. Q-TWiST analysis of patients receiving temsirolimus or interferon alpha for treatment 
of advanced renal cell carcinoma.

Pharmacoeconomics 28, 577-584 (2010).

35 Dutcher JP, de Souza P, McDermott D et al. Effect of temsirolimus versus interferon-alpha on outcome of patients with advanced renal cell carcinoma of different tumor histologies. Med. Oncol. 26, 202-209 (2009).

36 Bellmunt J, Dutcher J. Targeted therapies and the treatment of non-clear cell renal cell carcinoma. Ann. Oncol. 24, 1730-1740 (2013).

37 Logan T, McDermott D, Dutcher J et al. Exploratory analysis of the influence of nephrectomy status on temsirolimus efficacy in patients with advanced renal cell carcinoma and poor-risk features. Presented at: The 2009 Genitourinary Cancers Symposium. Orlando, FL, USA, 26-29 February 2009 (Abstract 281).

38 Mallick R, Chen J. Predictors of survival in patients with advanced renal cell carcinoma who received first line treatment with temsirolimus, interferon-alpha or combination temsirolimus/interferon alpha. Ann. Oncol. 19(Suppl. 8), Abstract 591P (2008).

39 Hutson TE, Escudier B, Esteban E et al. Randomized Phase III trial of temsirolimus versus sorafenib as second-line therapy after sunitinib in patients with metastatic renal cell carcinoma. J. Clin. Oncol. 32, 760-767 (2014).

- The Phase III INTORSECT trial compared the efficacy and safety of temsirolimus with that of sorafenib in mRCC patients who had received first-line sunitinib.

40 Woike M, Bergmann L, Steiner T et al. Evaluation of safety, tolerability and activity of temsirolimus in patients (pts) with advanced or metastatic renal cell carcinoma $(\mathrm{a} / \mathrm{mRCC})$ in routine clinical practice. Poster presented at: The 2014 European Society for Medical Oncology (ESMO) Congress. Madrid, Spain, 26-30 September 2014 (Abstract 850P).

41 Guida F, Santoni M, De Giorgi M et al. Poor risk metastatic Renal Cell Carcinoma (mRCC) patients are not a homogeneous group: a new stratificating model in the era of targeted therapy. Ann. Oncol. 25(Suppl. 4), iv280-iv304 (2014).

42 Bellmunt J, Szczylik C, Feingold J et al. Temsirolimus safety profile and management of toxic effects in patients with advanced renal cell carcinoma and poor prognostic features. Ann. Oncol. 19, 1387-1392 (2008).

43 Hutson TE, Figlin RA, Kuhn JG et al. Targeted therapies for metastatic renal cell carcinoma: an overview of toxicity and dosing strategies. Oncologist 13, 1084-1096 (2008).

44 Negrier S, Gravis G, Perol D et al. Temsirolimus and bevacizumab, or sunitinib, or interferon alfa and bevacizumab for patients with advanced renal cell carcinoma (TORAVA): a randomised Phase 2 trial. Lancet Oncol. 12, 673-680 (2011).

45 Hainsworth JD, Spigel DR, Burris HA et al. Phase II trial of bevacizumab and everolimus in patients with advanced renal cell carcinoma. J. Clin. Oncol. 28, 2131-2136 (2010).

46 Ravaud A, Barrios CH, Alekseev B et al. RECORD-2: Phase II randomized study of everolimus and bevacizumab versus interferon $\alpha-2 \mathrm{a}$ and bevacizumab as first-line therapy in patients with metastatic renal cell carcinoma. Ann. Oncol. 26(7), 1378-1384 (2015).

47 Rini BI, Bellmunt J, Clancy J et al. Randomized Phase III trial of temsirolimus and bevacizumab versus interferon alfa and bevacizumab in metastatic renal cell carcinoma: INTORACT trial. J. Clin. Oncol. 32, 752-759 (2014).

- $\quad$ The Phase III INTORACT study compared temsirolimus plus bevacizumab versus bevacizumab plus IFN- $\alpha$ in first-line mRCC patients.

48 Flaherty KT, Manola JB, Pins M et al. BEST: a randomized Phase II study of vascular endothelial growth factor, RAF kinase, and mammalian target of rapamycin combination targeted therapy with bevacizumab, sorafenib, and temsirolimus in advanced renal cell carcinoma-a trial of the ECOG-ACRIN Cancer Research Group (E2804). J. Clin. Oncol. 33(21), 2384-2391 (2015).

49 Clinical Trials database: NCT00782275. https://clinicaltrials.gov

50 Clinical Trials database: NCT01264341. https://clinicaltrials.gov

51 Novartis. Novartis Europharm Limited. Afinitor $^{\circledR}$ (everolimus) Summary of Product Characteristics (2014). www.medicines.org.uk

52 Escudier BJ, Abadie-Lacourtoisie S, Blanc E et al. Efficacy of everolimus in poor risk patients: result of the POORTOR study. J. Clin. Oncol. 33(Suppl. 7), Abstract 488 (2015).

53 Motzer RJ, Hutson TE, Tomczak P et al. Overall survival and updated results for sunitinib compared with interferon alfa in patients with metastatic renal cell carcinoma. J. Clin. Oncol. 27, 3584-3590 (2009).

54 Gore ME, Szczylik C, Porta C et al. Final results from the large sunitinib global expanded-access trial in metastatic renal cell carcinoma. Br. J. Cancer 113(1), 12-19 (2015).

55 Heng DY, Elson P, Golshayan AR et al. A retrospective multicenter study of MSKCC poor-prognosis patients with metastatic renal cell carcinoma (mRCC) treated with sunitinib. J. Clin. Oncol. 26(15 Suppl.), Abstract 16057 (2008).

56 Bamias A, Tzannis K, Beuselinck B et al. Development and validation of a prognostic model in patients with metastatic renal cell carcinoma treated with sunitinib: a European collaboration. Br. J. Cancer 109, 332-341 (2013).

57 Michaelson MD, Zhu AX, Ryan DP et al. Sunitinib in combination with gemcitabine for advanced solid tumours: a Phase I dose-finding study. Br. J. Cancer 108, 1393-1401 (2013).

58 McKay RR, Choueiri TK, Werner L et al. A Phase II trial of sunitinib and gemcitabine in sarcomatoid and/or poor-risk patients with metastatic renal cell carcinoma. J. Clin. Oncol. 33(Suppl. 7), Abstract 408 (2015).

59 Motzer RJ, Escudier B, Tomczak P et al. Axitinib versus sorafenib as second-line treatment for advanced renal cell carcinoma: overall survival analysis and updated results from a randomised Phase 3 trial. Lancet Oncol. 14, 552-562 (2013).

-• The Phase III AXIS trial compared second-line axitinib versus sorafenib in mRCC patients, a third of whom were categorized as poor risk.

60 Escudier B, Bellmunt J, Negrier $S$ et al. Phase III trial of bevacizumab plus interferon alfa-2a in patients with metastatic renal cell carcinoma (AVOREN): final analysis of overall survival. J. Clin. Oncol. 28, 2144-2150 (2010).

61 Rini BI, Halabi S, Rosenberg JE et al. Bevacizumab plus interferon alfa compared with interferon alfa monotherapy in patients with metastatic renal cell carcinoma: CALGB 90206. J. Clin. Oncol. 26, 5422-5428 (2008).

62 Rini BI, Halabi S, Rosenberg JE et al. Phase III trial of bevacizumab plus interferon alfa versus interferon alfa monotherapy in patients with metastatic renal cell carcinoma: final results of CALGB 90206. J. Clin. Oncol. 28, 2137-2143 (2010).

63 Motzer RJ, Nosov D, Eisen T et al. Tivozanib versus sorafenib as initial targeted therapy for patients with metastatic renal cell carcinoma: results from a Phase III trial. J. Clin. Oncol. 31, 3791-3799 (2013). 
64 Choueiri TK, Escudier B, Powles T et al. Cabozantinib versus everolimus in advanced renal-cell carcinoma. $N$. Engl. J. Med. 373(19), 1814-1823 (2015).

65 Clinical Trials databse: NCT01392183. http://clinicaltrials.gov/show/NCT01392183

66 Amin A, Dudek A, Logan T et al. Prolonged survival with personalized immunotherapy (AGS-003) in combination with sunitinib in unfavorable risk metastatic RCC (mRCC). J. Clin. Oncol. 31(Suppl. 6), Abstract 357 (2013).

67 Clinical Trials database: NCT01582672. http://clinicaltrials.gov/show/NCT01582672

68 Bailey A, McDermott DF. Immune checkpoint inhibitors as novel targets for renal cell carcinoma therapeutics. Cancer J. 19, 348-352 (2013).

69 Escudier B, Albiges L, Sonpavde G. Optimal management of metastatic renal cell carcinoma: current status. Drugs 73, 427-438 (2013).

70 Motzer RJ, Rini BI, McDermot DF et al. Nivolumab for metastatic renal cell carcinoma (mRCC): Results of a randomized, dose-ranging Phase II trial. Presented at: The 50th Annual Meeting of the American Society of Clinical Oncology (ASCO). Chicago, IL, USA, 30 May-3 June 2014 (Abstract 5009).

71 Motzer RJ, Rini BI, McDermot DF et al. Nivolumab for metastatic renal cell carcinoma: results of a randomized Phase II trial. J. Clin. Oncol. 33, 1430-1437 (2015).

72 Motzer RJ, Escudier B, McDermott DF et al. Nivolumab versus everolimus in advanced renal-cell carcinoma. N. Engl. J. Med. 373(19), 1803-1813 (2015).

73 Motzer RJ, Hutson TE, Cella D et al. Pazopanib versus sunitinib in metastatic renal-cell carcinoma. N. Engl. J. Med. 369, 722-731 (2013).

74 Choueiri TK, Figueroa DJ, Fay AP et al. Correlation of PD-L1 tumor expression and treatment outcomes in patients with renal cell carcinoma receiving sunitinib or pazopanib: results from COMPARZ, a randomized controlled trial. Clin. Cancer Res. 21, 1071-1077 (2015).

75 Clinical Trials databse: NCT01521715. http://clinicaltrials.gov/show/NCT01521715

76 Sanchez A, Domínguez RG, De Velasco G et al. Pazopanib in metastatic renal carcinoma $(\mathrm{mRC})$ : Experience of 31 centers in Spain in first, second, third, or subsequent lines in daily clinical practice. J. Clin. Oncol. 31(Suppl.), Abstract e15609 (2013).

77 Figlin RA, Kaufmann I, Brechbiel J. Targeting PI3K and mTORC2 in metastatic renal cell carcinoma: new strategies for overcoming resistance to VEGFR and mTORC1 inhibitors. Int. J. Cancer 133, 788-796 (2013).

78 Bendell JC, Rodon J, Burris HA et al. Phase I, dose-escalation study of BKM120, an oral panclass I PI3K inhibitor, in patients with advanced solid tumors. J. Clin. Oncol. 30, 282-290 (2012).

79 Grana B, Burris HA, Rodon Ahnert J et al. Oral PI3 kinase inhibitor BKM120 monotherapy in patients (pts) with advanced solid tumors: an update on safety and efficacy. 2011 ASCO Annual Meeting. J. Clin. Oncol. 29(Suppl.), Abstract 3043 (2011).

80 Burris H, Rodon J, Sharma S et al. First-inman Phase I study of the oral dual PI3K and mTORC1/2 inhibitor BEZ235 in patients with advanced solid tumors. J. Clin. Oncol. 28, Abstract 3005 (2010).

81 Powles T, Oudard S, Escudier BJ et al. A randomized Phase II study of GDC-0980 versus everolimus in metastatic renal cell carcinoma (mRCC) patients (pts) after VEGF-targeted therapy (VEGF-TT). J. Clin. Oncol. 32, Abstract 4525 (2014).

82 Powles T, Wheater M, Din O et al. A randomised Phase 2 study of AZD2014 versus everolimus in patients with VEGF-refractory metastatic clear cell renal cancer. Eur. Urol. doi:10.1016/j.eururo.2015.08.035 (2015) (Epub ahead of print).

83 Flanigan RC, Salmon SE, Blumenstein BA et al. Nephrectomy followed by interferon alfa-2b compared with interferon alfa- $2 b$ alone for metastatic renal-cell cancer. $N$. Engl. J. Med. 345, 1655-1659 (2001).

84 Mickisch GH, Garin A, van Poppel H et al. Radical nephrectomy plus interferon-alfabased immunotherapy compared with interferon alfa alone in metastatic renal-cell carcinoma: a randomised trial. Lancet 358, 966-970 (2001)

85 Warren M, Venner PM, North S et al. A population-based study examining the effect of tyrosine kinase inhibitors on survival in metastatic renal cell carcinoma in Alberta and the role of nephrectomy prior to treatment. Can. Urol. Assoc. J. 3, 281-289 (2009).

86 Kutikov A, Uzzo RG, Caraway A et al. Use of systemic therapy and factors affecting survival for patients undergoing cytoreductive nephrectomy. BJU. Int. 106, 218-223 (2010).

87 Jonasch E, Wood CG, Matin SF et al. Phase II presurgical feasibility study of bevacizumab in untreated patients with metastatic renal cell carcinoma. J. Clin. Oncol. 27, 4076-4081 (2009).
88 Abel EJ, Culp SH, Tannir NM et al. Primary tumor response to targeted agents in patients with metastatic renal cell carcinoma. Eur. Urol. 59, 10-15 (2011).

89 Clinical Trials database: NCT00930033. http://clinicaltrials.gov/show/NCT00930033

90 Clinical Trials database: NCT01099423. http://clinicaltrials.gov/show/NCT01099423

91 Cho D, Signoretti S, Dabora S et al. Potential histologic and molecular predictors of response to temsirolimus in patients with advanced renal cell carcinoma. Clin. Genitourin. Cancer 5, 379-385 (2007).

92 Fay AP, Kwiatkowski DJ, Gray KP et al. Activating genomic mutations in the mTOR pathway to predict responses to everolimus and temsirolimus in patients with metastatic renal cell carcinoma (mRCC): results from a large multi-institutional cohort. J. Clin. Oncol. 33(Suppl.), Abstract 4519 (2015).

93 Figlin RA, de Souza P, McDermott D et al. Analysis of PTEN and HIF-1alpha and correlation with efficacy in patients with advanced renal cell carcinoma treated with temsirolimus versus interferon-alpha. Cancer 115, 3651-3660 (2009).

94 Di Napoli A, Signoretti S. Tissue biomarkers in renal cell carcinoma: issues and solutions. Cancer 115(10 Suppl.), 2290-2297 (2009).

95 Armstrong AJ, George DJ, Halabi S. Serum lactate dehydrogenase predicts for overall survival benefit in patients with metastatic renal cell carcinoma treated with inhibition of mammalian target of rapamycin. J. Clin. Oncol. 30, 3402-3407 (2012).

96 Nallari A, Karrison T, Rosner GL et al. Fasting glucose and triglycerides as biomarkers of mTOR inhibition, evidence of a categorical response. J. Clin. Oncol. 28(Suppl. 15s), Abstract 3091 (2010).

97 Lee CK, Marschner IC, Simes RJ et al. Increase in cholesterol predicts survival advantage in renal cell carcinoma patients treated with temsirolimus. Clin. Cancer. Res. 18, 3188-3196 (2012).

98 Dienstmann R, Brana I, Rodon J et al. Toxicity as a biomarker of efficacy of molecular targeted therapies: focus on EGFR and VEGF inhibiting anticancer drugs. Oncologist 16, 1729-1740 (2011).

99 White DA, Camus P, Endo $\mathrm{M}$ et al. Noninfectious pneumonitis after everolimus therapy for advanced renal cell carcinoma. Am. J. Respir. Crit. Care Med. 182, 396-403 (2010).

100 Maroto JP, Hudes G, Dutcher JP et al. Drug-related pneumonitis in patients with 
advanced renal cell carcinoma treated with temsirolimus. J. Clin. Oncol. 29, 1750-1756 (2011).
101 Dabydeen DA, Jagannathan JP, Ramaiya N et al. Pneumonitis associated with mTOR inhibitors therapy in patients with metastatic renal cell carcinoma: incidence, radiographic findings and correlation with clinical outcome. Eur. J. Cancer 48, 1519-1524 (2012). 